\title{
Aspectos Epidemiológicos de la Prematurez
}

\author{
Drs. Ramiro Molina C." Jorge López* Hugo Muñoz**
}

La Prematurez es un problema de salud, que enfrenta tanto el obstétra como el pedjatra y naturalmente es una gituación aflictiva en extremo para los padres.

Los esfuerzos para la recuperación de los niños prematuros dependen principalmente de los avances y calidad del manejo pediátrico. El obstétra juega un papel importante en algunas áreas de su especialidad como la elección de la adecuada anestesia en un trabajo de parto prematuro, el adecuado monitoreo fetal y el criterio para manejar situeciones y condiciones obstétricas específicas en un parto premoturo o amenaza del mismo.

Pare el obstétra es importante anticiperse al parto prematuro y para ello es indispensable conocer sus causas y así tener conductas activas que incidan en la disminución de la frecuencia del fenómeno. Sólo con fines didácticos es necesario que nos pregunte-

\footnotetext{
*Departamento de Obatetricia y Gimecologis y Departamento de Salud Pública. Facultad de Medicina Norte.

* Pediatra, Departarnento de Salud Pública, Faculad Medicina Norte, U. de Chile.
}

mos äcuáles causas de prematurjad puede ger prevenidas o tratadas? ¿Qué medidas deberemos tomar para asegurar las mejores condiciones post parto a un niño que no ha alcanzado su desarrollo intrauterino adecuado? ¿Qué conductas obstétricas activas son negativas para un niño prematuro? ¿Cuándo se justifica interrumpir un embarazo en forma prematura ya sea por indicaciones médicas generales u obstétricas?

¿Cuándo se justifica detener o no un trabajo de parto prematuro? ¿Cuáles son los factores que inciden en la mejor edad gestacional para interrumpir un embarazo?

Para responder estas preguntas es necesario, por una parte, conocer la magnitud del problema, sus causas y los factores asociados como así también las técnicas y disponibilidad de recursos y organización de ellos.

Sin embargo, a pesar de todo, muchas causas quedarán fuera de los esfuerzos terapéuticos, los cuales se ubican principalmente en las condicionantes socioeconómicas o de estabilidad emocional y psicológica de la madre. 


\section{DEFINICION}

Antes de entrar en este acápite un tanto problemático, recordemos en general los cambios en peso y tamaño que muestra un niño desde su concepción.
Es el crecimiento en el último trimestre el que, por último, influenciará las posibilidades de Bobrevida, $y$ será este aumento esperado en su vida intrauterina lo que debemos tever giempre presente.

Tabla N. ${ }^{\circ} 1$

Cambios de peso y tamaño del feto según trimestre de Embarazo*

$$
\begin{array}{ccc}
\text { Peso en } \\
\substack{\text { \% de peso que } \\
\text { sumenta }} & \begin{array}{c}
\text { Longitud } \\
\text { en cms. }
\end{array} & \begin{array}{r}
\text { \% de longitud } \\
\text { que aumenta }
\end{array}
\end{array}
$$

\begin{tabular}{lcccr}
\hline Concepción & 0,000004 & & 0.014 & \\
Fin primer trimestre & 14 & 300.000 .000 & 7 & 50.000 \\
Fin segundo trimestre & 700 & 5.000 & 35 & 400 \\
Fin del tercer & 3.500 & 400 & 50 & 50 \\
trimestre & & & & \\
\hline
\end{tabular}

"Tomado de Harper "Preventive Pediatrics" Child Health and Development, New York, Appleton-Century-Crofts, 1962.

En general, es también claso que el peso al nacimiento depende de la duración de la gestación y estas dos variables son las que durante mucho tiempo has influido para tener una definición universaimente eceptada.

Revisemos una serie de términos principalmente desde un punto de vista del producto, objeto último de este análigis.

RECIEN NACIDO PREMATURO: R.N. cuyo peso al nacer es de $2.500 \mathrm{grs}$. o menos, independientemente de su edad gestacional.

RECIEN NACIDO PRE-TERMINO: R.N. producto de una gestación de 37 semanas o menos. RECIEN NACIDO PRETERMINO ADECUADO A SU EDAD GESTACIONAL (R.N. Pr. A.E.G.): Es aquel R.N. de 37 semanas o menos cuyo peso al nacer se encuentra entre el percentil 10 y 90 de la curva de crecimiento intrauterino.

RECIEN NACIDO PRE-TERMINO PEQUEÑO PARA SU EDAD GESTACIONAL (R.N. Pr. P.E. G.): Es aquel R.N. de 37 semanas o menos de gestación cuyo peso al nacer se encuentra bajo el percetil 10 de la curva de crecimiento intrauterino.

Varios autores han determinado curvas de crecimiento ponderal intrauterino, pero la más usada en nuestro medio es la curva de Lubchenco."

RECIEN NACIDO DE BAJO PESO DE NACIMIENTO: Para algunos autores es equivalente al $R$. N. prematuro.

RECIEN NACIDO DE PESO INSUFICIENTE:

Algunos autores consideran en este rubro al R.N. cuyo peso al nacer fluctúa entre 2.500 y $3.000 \mathrm{gra}$., independientemente de su edad gestacional.

RECIEN NACIDO DE MUY BAJO PESO: Es aquel R.N. cuyo peso al nacer es menor de 1.250 ó $1.500 \mathrm{grs}$. al nacer.

INMATURIDAD NO CALIFICADA: Niños que nacen vivos de 2.500 ó menos y cuya causa de muerte se desconoce. (N. 777 según Clasificación Internacional de Enfermedady.

PARTO PREMATURO: Es aquel que ocurre entre la $27^{2}$ y $38^{a}$ semanas de gestación.

AMENAZA DE PARTO PREMATURO: Aquel embarazo entre la $27^{a}$ y $38^{\circ}$ semanas de gestación que inicia contracciones uterinas similares a la del trabajo de parto.

DESNUTRIDO FETAL: Recién nacido con gignos de malnutricion fetal, (enflaquecido, ojos abjertos, piel macerada o descamada, impregnación de meconio, etc.) con peso al nacer frecuentemente bajo percentil 10 para su edad gestacional según la curva de crecimiento intrauterino.

\section{MAGNITUD DEL, PROBLEMA}

La incidencia de] fenómeno a nivel nacional tanto en el extranjero como en Chile es mal conocida pues el dato de peso al nacimiento y edad del embarazo no es registrada habitualmente en los Certificados de Parto o Certificados de Nacimiento. De tal modo que esta información se limita a Regiones, Areas geográficas o Maternidades y se proyecta esta información al resto de la población.

Además, los criterios para definir prematuridad no son homogéneos y por lo tanto la comparabilidad de un país a otro no es exacta. 
La mayoria de las publicaciones adoptan el criterio de peso del R.N. de $2.500 \mathrm{gr}$. y menos para definir prematuridad lo cual abarca seguramente a Recién
Nacidos con desnutrición fetal. Sin embargo, este error en último término juega en función de la seguridad de estos últimos.

Tabla $N^{0}{ }^{\circ} 2$

Frecuencia de Prematurez en diferentes partes de] mundo

\begin{tabular}{|c|c|c|c|}
\hline AUTOR & ORIGEN & $\begin{array}{r}\text { TOTAL DE } \\
\text { NACIMIENTOS }\end{array}$ & $\begin{array}{r}\% \text { DE } \\
\text { PREMATLROS }\end{array}$ \\
\hline Smith & Bostón & - & 6.8 \\
\hline Steer & Ciudad de Nueva York & 44.124 & 9.0 \\
\hline Rouchy y Taureau & Francia & - & 6.0 \\
\hline Kaijger y Frankel & Suecia & 8.284 & 5.6. \\
\hline Taback & Baltimore & 9.709 & 8.9 \\
\hline Baba y Nakamura & Japón & 3.100 & 11.2 \\
\hline Swyer & Canadá & - & 7.0 \\
\hline Arneaud y Bras & Jamaica & 2.637 & 12.5 \\
\hline Douglas & Gran Bretaña & 13.257 & 6.5 \\
\hline Kubát y Polácek & Checoslovaquia & 5.455 & 10.5 \\
\hline Bain y al. & Estados Unidos & - & 6.1 \\
\hline Moller & Suecia & - & 6.2 \\
\hline Sanford y al. & Chicago & - & 8.0 \\
\hline Songeo & Filipinas & - & 18.3 \\
\hline Loria Cortés & Cuba & 7.353 & 10.1 \\
\hline Selfa & España & - & 5.0 \\
\hline Reyes-Agcaoili y al. & Filipinas & 8.859 & 10.5 \\
\hline Baumgartner y al. & Nueva York & - & 8.0 \\
\hline de Araujo & Brasil & 一 & 7.0 \\
\hline Rosenfield y al. & Minnessota & - & 7.0 \\
\hline Lechtig y al. & Colorado & - & 30.8 \\
\hline Merger y Haquin & Francja & 4.708 & 7.05 \\
\hline Gross & Viena & 13.190 & 7.8 \\
\hline Lewis & Inglaterra y Gales & - & 7.0 \\
\hline Noack y Opitz & Alemania & 57.426 & 7.55 \\
\hline Folsome y al. & Ciudad de Nueva York & 2.400 & 6.3 \\
\hline Hirschl y al. & Nueva York & 13.334 & 4.6. \\
\hline Van Geldersen y al. & Holanda & - & 3.5 \\
\hline Parmelee & Los Angeles & I.019 & 6.3 \\
\hline Russell y Betts & Tulsa, Olda. & 20.331 & 6.8 \\
\hline Halevi & Israel & - & 4.7 a 7.1 \\
\hline Bogin y Edgar & Bridgeport. Conn. & - & 7.0 \\
\hline Szenas & Suiza & - & 6.9 \\
\hline Nesbitt & Estados Unidos & - & 6.1 \\
\hline Javert & Estados Unidos & 30.788 & 5.7 \\
\hline Adam & Australia & - & 5.0 a 10 \\
\hline Hilfrich & Alemania & - & 7.1 \\
\hline
\end{tabular}

*Tomado de Cavenagh “Prerantury and the Obatetrician”. Appleton Century-Croft s. Educational Division. New York, 1969.

Según esta revieión, la frecuencia varía de $3.5 \%$ en Holanda a 18.3\% en Filipinas. Hace excepción la incidencia de $30.8 \%$ reportado por Lechtig y colabo- raderes que correaponde a un lugar de $1.609 \mathrm{~m}$ sobre el nivel del mar en el Estado de Colorado.

En nuestra Maternidad del Hospital J.J. Aguirre 
la incidencia del niño de bajo peso al nacer definidos como nacidos vivos de $2.500 \mathrm{gr}$ y menog independiente de la edad gestacional ba variado de $9.3 \%$ a $12.3 \%$ del total de nacidos vivos, En la Tabla N. ${ }^{\circ} 3 \mathrm{se}$ aprecia el detalle de estus cifras desde 1970 a 1978.

Tabla N. ${ }^{9} 3$

Incidencia de niños de bajo peso.

Maternidad Hospital José Joaguín Aguirre *

(1970-1978)

\begin{tabular}{rrrr} 
Año & $\begin{array}{r}\text { Total de } \\
\text { Nac. vivos }\end{array}$ & $\begin{array}{r}\text { Nacidos de } \\
2.500 \mathrm{~g}\end{array}$ & \% $(-)$ \\
\hline 1970 & 9.307 & 1.016 & 10.8 \\
1971 & 10.709 & 1.082 & 10.1 \\
1972 & 11.330 & 1.372 & 12.1 \\
1973 & 11.153 & 1.197 & 10.7 \\
1974 & 11.103 & 1.035 & 9.3 \\
1975 & 7.394 & 910 & 12.3 \\
1976 & 6.998 & 816 & 11.7 \\
1977 & 5.680 & 627 & 11.0 \\
1978 & 2.934 & 300 & 10.2 \\
\hline
\end{tabular}

-Anuarioa Estadíticos Hodpital J.J. Aquirre.

Tomando como referencias estos índices y proyectándolos a nivel nacional, podemos estimar que en 1977 en Chile hubo $\mathbf{2 4 0 . 4 6 3}$ nacidos vivos, de los cuales aproximadamente entre 22.363 (9.3\%) a 29.577 (12.3\%) fueron niños de bajo peso.

En la Maternidad del Hospital José Joaquín Aguirre ae tabularon los datos de recién nacidos según la edad gestacional al parto dando los siguientes resul. tados.

\section{Tabla N. ${ }^{\circ} 4$}

Recién Nacidos Vivos según Edad Gestacional. Maternidad J.J. Aguirre. 1977.

\begin{tabular}{lrr} 
Calificación & $N$. & $\%$ \\
Pre-término & 1.013 & 17.8 \\
Término & 3.438 & 60.6 \\
Post-término & 34 & 0.6 \\
No consignado & 1.195 & 21,0 \\
\hline TOTAL & 5.690 & 100.0
\end{tabular}

Aungue estas cifras están muy influenciades por los no consignados una proyección global a Chile, nos da que para $1977,40.879$ nacidos vivos pueden haber sido de pre-término.
Por último, una estimación de Lechti ${ }^{8}$ nos muestra que la incidencia de niños de $2.500 \mathrm{~g}$. o menos, varía de $4 \%$ en la Unión Soviética a $21 \%$ en Asia (Tabla N. ${ }^{\circ}$ ).

\section{Table N. ${ }^{\circ} 5$}

Incidencia de niños con $2.500 \mathrm{~g}$ o menos según

$$
\text { Regiones* }
$$

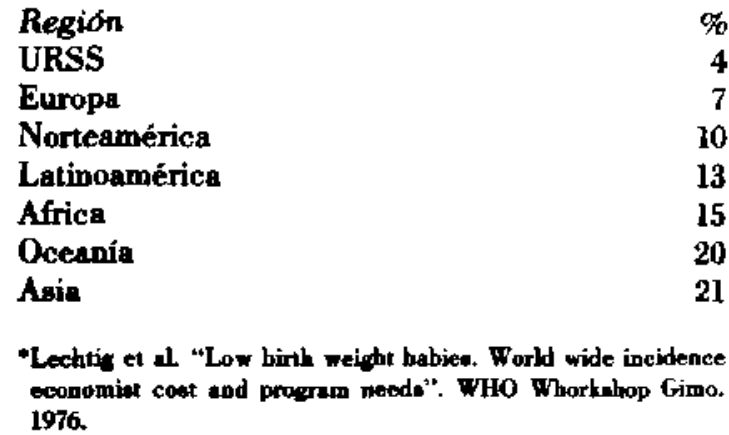

\section{MORBILIDAD}

La Morbilided asociada a la prematurez puede ser precoz o tardía.

Su alta labilidad a las enfermedades se deben principalmente a la inmadurez orgánica y funcional de aus sintemas.

1. Aparato Respiratorio: Tendencia al síndrome de dificultad respiratoria idiopático.

2. Aparato Digeativo: Dificultad para succionar, baja tolerancia gástrica, dificultad de metabolización, tránsito intestinal.

3. Sistema Nerviogo Central: Crisis de apnea, difcultad para auccionar y deglutir, etc.

4. Función Hepática: Tendencia a la Ictericia por falla en la conjugación, hipaglicemia y manifestaciones hemorrágicas por déficit de Vit. $K$, fibrinógenos y protrombina.

5. Siotema Hematopoyético: Anemia hipocroma por falta principalmente de Fe.

6. Termorregulación: Falla para mantener temperatura corporal constante y normal: gran superficie corporal en relación al peso, déficit de tejido adiposo y alándulas sudoriparas mal deasrolladas; además existe inmadurez centrul.

7. Cardiovascular: Aumento de la fragilidad capilar que predispone a hemortagias.

8. Sistema Inmunitario que predispone a le alta incidencia a las infecciones: piel, entéricas, respiratorias y sepeis.

9. Alta frecuencia de malformaciones congénitas.

Un aspecto de extradordinaria importancia de analizar es la morbilidad del embarazo asociado a la prematurez. 
De un estudio en la Maternidad de] Hospital J.J. Azuirre se observó que la infección del tracto urinario y la colestagia intrahepática fueron las que acumularon mayor proporción de niñoe de $2.500 \mathrm{~g}$. y menos.

En ambas el problema afecta más a las mujeres, probablemente por el déficit biológico de peso de nacimiento con respecto al varón.

\section{Tabla N. ${ }^{\circ} 6$}

Incidencia de niños de 2.500 g y menos según sexo y patología del emberazo

\begin{tabular}{lccl}
$\begin{array}{l}\text { Patología } \\
\text { Materna }\end{array}$ & $\begin{array}{c}\text { Varones } \\
\%\end{array}$ & $\begin{array}{c}\text { Mujeres } \\
\%\end{array}$ & Total \\
\hline $\begin{array}{l}\text { Inf. Tracto Urinario } \\
\text { Toxemia }\end{array}$ & $18.7^{* *}$ & $35.0^{* *}$ & $27.0^{* * *}$ \\
Colestasia intrah. & 10.5 & $22.2^{* *}$ & 17.0 \\
Diabetes & 7.9 & $13.0^{* *}$ & $14.8^{* *}$ \\
Otros & 0 & 22.2 & 10.0 \\
Normal & $18.9^{* *}$ & 18.3 & $19.3^{* *}$ \\
& 9.6 & 11.3 & 10.3 \\
& $* p$ & 0.05 de los normales.
\end{tabular}

"Rizzardini, M.; Muñoz, H.; Murillo, A.; Muñoz, F.; Mrazde, E.; “Almunus variablea anociadan de peso de nacimiento". Congreso de Pediatria. Autofacasta, 1979 (Por publicarse).

En nuestra Maternidad la morbilidad en el niño de pre-término comparada con el término durante 1977 se aprecia en la Tabla $N .^{\circ} 7$.

\section{Tabla N. ${ }^{\circ} 7$}

Morbilidad del nin̄o de término y pre-término Hospital J.J. Aguirre 1977.

(Tasa por 100 N.V.)

\begin{tabular}{lrrrr} 
Diagnóstico & \multicolumn{2}{c}{ Término } & & Pre-término \\
& N.0 & $\%$ & N. & $\%$ \\
Sindrome de dif. Regp. & 47 & 1.4 & 81 & 8.0 \\
Cianosis & 46 & 1.3 & 62 & 6.1 \\
Asfixia Neonatal & 52 & 1.5 & 30 & 3.0 \\
Sufrimiento fetal & 37 & 1.0 & 14 & 1.4 \\
Asfixia Recién Nacido & 15 & 0.4 & 9 & 0.9 \\
Aspiración del contenido & & & & \\
del conducto del parto & 6 & 0.2 & 3 & 0.3 \\
Enfermedad hemolítica & 18 & 0.5 & 3 & 0.3 \\
Malformaciones & 74 & 2.2 & 22 & 2.2 \\
\hline TOTAL N.V. & 3.438 & & 1.013 & \\
\hline
\end{tabular}

Los problemas relacionados con la patología de] Aparato Respiratorio bon fundamentales, siendo el síndrome de Dificultad Respiratoria el más impor- tante.

Ahora, si proyectamos estos hallazgos a nivel nacional se obtiene lo que muestra la Tabla $N .{ }^{\circ} 8$. 


\section{Tabla N. ${ }^{\circ} \mathbf{B}$}

Estimación de las morbilidades del Nin̄o de Pre-término en Chile 1977

\section{Diagnostico}

Sindrome de Dific. Respirat. Cinnosis

Asfixia Neonatal

Sufrimiento fetal

Asfixia del Recién Nacido

Aspiración del contenido del conducto del parto
El problema de la asfixia se puede abordar mediante la incidencia Apgar bajo 6 al minuto.

Tabla N. ${ }^{\circ} 9$

Incidencia de asfixia (Apgar $\leq 6$ ) según peso al nacer*

Peso \%

$3.270 \quad 2.500$ o menos $\quad 31.5$

2.494

1.226

De esta estimación se puede apreciar que un volumen de 8.000 niños o más necesitarán de técnicas ayanzadas de recuperación perinatal. Eate grupo de niños de alto riesgo necesariamente pasarán a ser un contingente de ls mortalidad neonatal si no se cuenta con la tecnología suficiente y normas claras de conducta.

$\begin{array}{lrl}2.501 \text { o más } & 7.6 & \text { p/0.01 } \\ \text { TOTAL } & 10.5\end{array}$

"Muñoz, H., y cols. "Variables asociadas al Teat de Apgar". Depto. Salud Públice 1978.

Prácticamente un tercio de los prematuros requieren atención inmediata y urgente por problemas de asfixia.

Las consecuencias tardias de la prematuridad o morbilidad tardía se ubican en el campo del retardo del desarrollo psicomotor del niño. Esta materia ha sido largamente informada y discutida, sin embargo, los seguimientos son difíciles de obtener y aún más los grupos controles, para sacar conclusiones.

Se estima que entre 15 y $20 \%$ de todos los niños con retardo mental corresponden a factores de asociación con patologías maternas durante el embarazo y prematuridad. ${ }^{6}$

Tabla N. ${ }^{\circ} 10$

Evalueción de la Mortalidad Neonatal por Inmaturidad no calificada Chile 1970-1977

Año Inmaturidad no calificada

\begin{tabular}{ccccc}
\hline & N. ${ }^{\circ}$ & $\begin{array}{c}\text { Tasa por } \\
1.000 \mathrm{~N} . \mathrm{V} .\end{array}$ & $\begin{array}{c}\text { \% de las muertes } \\
\text { neonatales }\end{array}$ & $\begin{array}{c}\text { N. }^{\circ} \text { estimado como } \\
\text { causa asociada }\end{array}$ \\
\hline 1970 & 807 & 3.1 & 18.4 & 4.917 \\
1971 & 775 & 2.8 & 17.1 & 4.646 \\
1972 & 701 & 2.5 & 11.9 & 4.837 \\
1973 & 629 & 2.3 & 10.1 & 4.470 \\
1974 & 482 & 1.8 & 8.5 & 4.142 \\
1975 & 413 & 1.6 & 9.3 & 3.821 \\
1976 & 323 & 1.3 & 6.9 & 3.509 \\
1977 & 211 & 0.9 & 4.9 & 3.022 \\
\hline
\end{tabular}

Sin la menor duda que la tendencia es hacia la disminución, en parte por mejor diagnóstico que especifica la causa de muerte.

Sin embargo, en el estudio de la Dra. Ruth Puffer ${ }^{13}$ se demostró que el $60 \%$ de la tasa de mortalidad neonatal estaba relacionada con el diagnóstico de Prematurez en forma asociada, o como causa básica.

Con esta estimación se puede observar las muertes de neonatos asociadas a prematuridad. Ciertamente que el panorama cambia fuertemente.

La mortalidad del prematuro es intrahospitalari; en su mayoria siendo más frecuente en el hombre qut la mujer, se acumula en la prímera semana y de ést: en el primer día (15\%). A su vez en la primera hora do vida se concentran las muertes neonatales. ${ }^{14}$ 
En la Tabla 11 se aprecia la mortalidad neonatal por peso y sexo.

Tabla N. ${ }^{\circ} 11$

Mortalidad neonatal según peso y sexo

del recién nacido

(Maternidad J.J. Aguirre, 1977)

(Tasa por 1,000 )

\begin{tabular}{cccc}
\hline Peso & Hombres & Mujeres & Total \\
\hline $600-999$ & 1.000 & 800.0 & 892.4 \\
$1.000-1.249$ & 676.4 & 617.6 & 647.1 \\
$1.250-1.499$ & 344.8 & 298.3 & 327.6 \\
$1.500-1.749$ & 268.8 & 175.6 & 221.6 \\
$1.750-2.000$ & 129.0 & 102.7 & 114.8 \\
\hline Total & 314.0 & 259.5 & 290.2 \\
\hline
\end{tabular}

EJ otro aspecto importante de conocer se refiere a las causas de muerte del prematuro. En el recién nacido de 2.000 g y menos, observó que el Síndrome de Dificultad Respiratoria es la causa más importante de muerte y se extiende hasta la mitad de la tercera semana de vida."

Las infecciones como causa de muerte se inician hacia fines de la primera semana.

\section{Prevencion:}

La prevención de la prematurez tiene varios niveles que se pueden describir en el siguiente cuadro esquemático.
Nivel

Acciones

\section{Protección Específica} 3. Diagnóstico
Precoz

a) Atención médica para detectar mujeres de alto riesgo.

b) Detección de patologias asociadas a parto prematuro.

c) Detección de patologías ginecológicas asociadas a parto prematuro.
a) Parto institucional.
b) Monitorización fetal.
c) Manejo del parto prematuro.
d) Atención inmedjata del re- cién nacido (personal-técni- co).
e) Atención especializada del R.N. prematuro. Oportuno

5. Rehabilitación a) Control de crecimiento y desarrollo.
b) Consultorio de seguimiento de prematuros.

c) Tratamiento y prevención de morbilidad.

\section{Evaluacion:}

El tratamiento del fenómeno prematurez, en forma racional, es prevenir las causas que lo provocan de las cuales muchas se encuentran en el terreno de lo desconocido y en último término nos reducimos a tratamiento de las patologias que dan como consecuencia un parto prematuro.

El segundo nivel es el tratamiento del parto prematuro y el manejo de la embarazada en tal situación.

EJ tercer nivel es la conducta obstétrica-pediátrica frente a un trabajo de parto prematuro y la tecnología necesaria para su monitorización.

El cuarto nivel es el manejo pediátrico del prematuro.

No cabe duda que en los dos últimos niveles es necesario contar con los medios humanos y materiales adecuados.

En una evaluación hecha en $1976,{ }^{2}$ en 25 áreas de salud de Chile, se encontró que los niveles de acreditación alcanzados por los servicios de recién nacidos y prematuros fueron los más bajos en eficiencia en relación a diferentes servicios matemo-infantiles, alcanzando un $57 \%$ de la eficiencia, log rangos variaron de $21 \%$ en Ancud a $81 \%$ en Osomo.

En los diferentes rubros analizados en los servicios de recién nacidos y prematuros la distribución de eficiencia alcanzada fue: 


\section{Rubro}

$\%$ de

Efic.

Registros

86.3

Técnicas de atención

69.8

Coordinación

68.6

Equipo e instrumental

61.9

Personal

55.8

Planta física

46.9

Contenidos de salud del personal

32.9

Organización y Programación

Condiciones de efíciencie de los Servicios Materno-Infantiles 25 Areas. Peamib 1976. Ministerio de Salud.

Como es fácil observar las tecnologías de equipo e instrumental no son los únicos elementos deficitarios y justamente los rubros de contenidos de salud del personal y la organización y programación que son la base para un mejor rendimiento pueden aún ser mejorados. Estos últimos dependen directamente del interés de los directivos responsables y de la motivación en relación al problema del recién nacido y prematuro.

\section{RECOMENDACIONES}

Para evaluar en mejor forma la atención del prematuro, es necesario señalar algunos puntos críticos:

l. Acordar terminologías comunes.

2. Buscar mejor indicadores de medición.

a) Incidencia de parto prematuro.

b) Incidencia de patologias obstétricas.

3. Auditorías médicas para revisar normas obstétricas-pediátricas.

4. Acreditación regular de eervicios.

5. Evaluar rendimiento de programas y técnicas de servicios.

\section{REFERENCIAS}

'Cavanagh. "Prematury and Obstetrician". Appletan-CenturyCrofts. Educational Division, New York, 1969.

${ }^{2}$ Condiciones de eficiencia de los Servicios Materno-Infantilea 25 Areas Pesmib. Minioterio de Salud, 1974.

${ }^{3}$ Dazzarolo, P. y S. Gonzalex. La prematurez en consultorio disIrilal. Análisis de al gunos finctoren determinantes, caracteristicas y evolución de 63 nióos controlados en el Coneultorio Independencia. Pediatria, 11: 289, 290, 1958.

${ }^{4}$ Fertilio y otros. Parto prematuro y feto de alto riesgo. XIV Con. greso Chileno Obstetricia y Ginecalogía, Sentiago, 1971.

${ }^{5}$ Galecio, Gr. y otros. Factores etiológicos menos frecuentee de] parto prematuno. Servicio Obstétrico Pediátrico, Matemidad del Salvador. XIV Congreso Chileno Obstetriciz y Ginecologia, Santiago, 1971.

"Harper. "Preventive Pediatrics", Child Health and Development, New York, Appleton. Century Crofls, 1962.

${ }^{7} \mathrm{Hemminki,} \mathrm{E.} \mathrm{et} \mathrm{ol.} \mathrm{Prevention} \mathrm{and} \mathrm{Treatment} \mathrm{of} \mathrm{Premanure} \mathrm{lo-}$ bour by Drugs: fleview of controlled clioical Trials. BR. Obet. Gynaecolo. 35 (6): 411-7. June 78 .

${ }^{8}$ Lechtig, et al. "Low birth weight babies. World Wide incidence, economic cost and program needs". W.H.O. Workshop Gimo., 1976.

${ }^{9}$ Lubchenco, L.; Hausman, C.; Dressler, H, and Bayd, E. "Intrauterine Growth as estimnted from live born. Birth weight deta at 24 to 42 weeke of geslation". Pediatrics, 32: 793, 1965.

10 Murioz, H. y cols. "Variables asociadas al Test de Aprar". Departamenta Salud Públice. Facultad Medicina Nonte, Universidad de Chile, 1978 (no publicado).

11 Muñoz y cols. "Sobrevida intrahospitalaria del recitn nocido de 2.000 g o menos". Rev. Chilena Pediatria, Vol. 1-6, 1978 .

12 Niebul, Jr. et al. "The pharmacologic inhibition of premature labor". Obs. Gynecol. Surv. 33 (8): 507.15, Aug. 1978.

${ }^{13}$ Puffer, Ruth; Serreno, Cartos. Estudio interamericano de mortalidad de la niñez. Publicaciones Cientificas N. ${ }^{\circ 262}$, OPS-OMS, 73.

${ }^{14}$ Rizzardini, M., Muñoz, H.; Murillo, A.; Muñoz, F.; Miranda, E. "Algunas variables arociadas al peso de nacimiento". Confreвo de Pediatría, Antofagasta, 1979 (por publicarae). 